\title{
Spectrum of the Sturm-Liouville operators with boundary conditions polynomially dependent on the spectral parameter
}

\author{
Nihal Yokus ${ }^{1 *}$ and Turhan Koprubasi ${ }^{2}$
}

\section{"Correspondence:}

nyokus@kmu.edu.tr

1 Department of Mathematics,

Karamanoglu Mehmetbey

University, Karaman, 70100, Turkey

Full list of author information is

available at the end of the article

\begin{abstract}
In this paper, we consider the operator $L$ generated in $L_{2}\left(\mathbb{R}_{+}\right)$by the Sturm-Liouville equation $-y^{\prime \prime}+q(x) y=\lambda^{2} y, x \in \mathbb{R}_{+}=[0, \infty)$, and the boundary condition $\left(\alpha_{0}+\alpha_{1} \lambda+\alpha_{2} \lambda^{2}\right) y^{\prime}(0)-\left(\beta_{0}+\beta_{1} \lambda+\beta_{2} \lambda^{2}\right) y(0)=0$, where $q$ is a complex-valued function, $\alpha_{i}, \beta_{i} \in \mathbb{C}, i=0,1,2$, and $\lambda$ is an eigenparameter. Under the conditions $q, q^{\prime} \in \mathrm{AC}\left(\mathbb{R}_{+}\right), \lim _{x \rightarrow \infty}|q(x)|+\left|q^{\prime}(x)\right|=0, \sup _{x \in \mathbb{R}_{+}}\left[e^{\varepsilon \sqrt{x}}\left|q^{\prime \prime}(x)\right|\right]<\infty, \varepsilon>0$, using the uniqueness theorems of analytic functions, we prove that $L$ has a finite number of eigenvalues and spectral singularities with finite multiplicities.
\end{abstract}

MSC: 34B08; 34B09; 34B24

Keywords: Sturm-Liouville equations; eigenparameter; eigenvalues; spectral singularities

\section{Introduction}

Let us consider the non-selfadjoint Sturm-Liouville operator $L_{0}$ generated in $L_{2}\left(\mathbb{R}_{+}\right)$by the differential expression

$$
l_{0}(y):=-y^{\prime \prime}+q(x) y, \quad x \in \mathbb{R}_{+},
$$

and the boundary condition $y^{\prime}(0)-h y(0)=0$, where $q$ is a complex-valued function and $h \in \mathbb{C}$. The spectrum and eigenfunction expansion of $L_{0}$ were investigated by Naimark [1]. In this study, the spectrum of $L_{0}$ is investigated and it is shown that it is composed of the eigenvalues, a continuous spectrum, and spectral singularities. The spectral singularities are poles of the resolvent which are embedded in the continuous spectrum and are not eigenvalues.

The effect of the spectral singularities in the spectral expansion of $L_{0}$ in terms of the principal functions has been investigated in [2-4].

The spectral analysis of the non-selfadjoint operator, generated in $L_{2}\left(\mathbb{R}_{+}\right)$by $(1.1)$ and the integral boundary condition

$$
\int_{0}^{\infty} A(x) y(x) d x+\alpha y^{\prime}(0)-\beta y(0)=0
$$

where $A \in L_{2}\left(\mathbb{R}_{+}\right)$is a complex-valued function, and $\alpha, \beta \in \mathbb{C}$, was investigated in detail by Krall $[5,6]$.

\section{Springer}

(c) 2015 Yokus and Koprubasi; licensee Springer. This is an Open Access article distributed under the terms of the Creative Commons Attribution License (http://creativecommons.org/licenses/by/4.0), which permits unrestricted use, distribution, and reproduction in any medium, provided the original work is properly credited. 
Some problems of spectral theory of differential and other types of operators with spectral singularities were also studied in [7-12].

Note that in all the above articles, the boundary conditions are independent of the spectral parameter.

In 1977, Fulton [13], considered the Sturm-Liouville equation with one boundary condition dependent on the spectral parameter and obtained asymptotic estimates of eigenvalues or eigenfunctions. Since 1977, one of such Sturm-Liouville equations with boundary condition dependent on the spectral parameter was discussed by a number of authors (see [14-21]).

Let $L$ denote the operator generated in $L_{2}\left(\mathbb{R}_{+}\right)$by

$$
\begin{aligned}
& -y^{\prime \prime}+q(x) y=\lambda^{2} y, \quad x \in \mathbb{R}_{+}, \\
& \left(\alpha_{0}+\alpha_{1} \lambda+\alpha_{2} \lambda^{2}\right) y^{\prime}(0)-\left(\beta_{0}+\beta_{1} \lambda+\beta_{2} \lambda^{2}\right) y(0)=0,
\end{aligned}
$$

where $q$ is a complex-valued function, $\alpha_{i}, \beta_{i} \in \mathbb{C}, i=0,1,2$, with $\left|\alpha_{2}\right|+\left|\beta_{2}\right| \neq 0$.

Differently from other studies in the literature, the specific feature of this paper, which is one of the articles having applicability in study areas such as physics, engineering, and mathematics, is the presence of the spectral parameter not only in the Sturm-Liouville equation but also in the boundary condition for a quadratic form.

In this article, we intend to investigate eigenvalues and the spectral singularities of the $L$, which has a finite number of eigenvalues and spectral singularities with a finite multiplicities, if the conditions

$$
\begin{aligned}
& q, q^{\prime} \in \mathrm{AC}\left(\mathbb{R}_{+}\right), \quad \lim _{x \rightarrow \infty}|q(x)|+\left|q^{\prime}(x)\right|=0, \\
& \sup _{x \in \mathbb{R}_{+}}\left[e^{\varepsilon \sqrt{x}}\left|q^{\prime \prime}(x)\right|\right]<\infty, \quad \varepsilon>0,
\end{aligned}
$$

hold, where $\mathrm{AC}\left(\mathbb{R}_{+}\right)$denotes the class of complex-valued absolutely continuous functions on $\mathbb{R}_{+}$.

\section{Jost solutions and Jost functions of $L$}

Let us suppose that

$$
\int_{0}^{\infty} x|q(x)| d x<\infty
$$

By $e(x, \lambda)$, we will denote the bounded solution of (1.2) satisfying the condition

$$
\lim _{x \rightarrow \infty} y(x, \lambda) e^{-i \lambda x}=1, \quad \text { for } \lambda \in \overline{\mathbb{C}}_{+}:=\{\lambda: \lambda \in \mathbb{C}, \operatorname{Im} \lambda \geq 0\} .
$$

The solution $e(x, \lambda)$ is called the Jost solution of (1.2). Under the condition (2.1), the solution $e(x, \lambda)$ has the integral representation [22, Chapter 3]

$$
e(x, \lambda)=e^{i \lambda x}+\int_{x}^{\infty} K(x, t) e^{i \lambda t} d t
$$


where the function $K(x, t)$ is the solution of the integral equation

$$
\begin{aligned}
K(x, t)= & \frac{1}{2} \int_{\frac{x+t}{2}}^{\infty} q(s) d s+\frac{1}{2} \int_{x}^{\frac{x+t}{2}} \int_{t+x-s}^{t+s-x} q(s) K(s, u) d u d s \\
& +\frac{1}{2} \int_{\frac{x+t}{2}}^{\infty} \int_{s}^{t+s-x} q(s) K(s, u) d u d s,
\end{aligned}
$$

and $K(x, t)$ is continuously differentiable with respect to its arguments. We also have

$$
\begin{aligned}
& |K(x, t)|<c w\left(\frac{x+t}{2}\right), \\
& \left|K_{x}(x, t)\right|,\left|K_{t}(x, t)\right| \leq \frac{1}{4}\left|q\left(\frac{x+t}{2}\right)\right|+c w\left(\frac{x+t}{2}\right),
\end{aligned}
$$

where $w(x)=\int_{x}^{\infty}|q(s)| d s$ and $c>0$ is a constant.

Let

$$
\begin{aligned}
& N^{+}(\lambda):=\left(\alpha_{0}+\alpha_{1} \lambda+\alpha_{2} \lambda^{2}\right) e^{\prime}(0, \lambda)-\left(\beta_{0}+\beta_{1} \lambda+\beta_{2} \lambda^{2}\right) e(0, \lambda), \quad \lambda \in \overline{\mathbb{C}}_{+}, \\
& N^{-}(\lambda):=\left(\alpha_{0}+\alpha_{1} \lambda+\alpha_{2} \lambda^{2}\right) e^{\prime}(0,-\lambda)-\left(\beta_{0}+\beta_{1} \lambda+\beta_{2} \lambda^{2}\right) e(0,-\lambda), \quad \lambda \in \overline{\mathbb{C}}_{-},
\end{aligned}
$$

where $\overline{\mathbb{C}}_{-}=\{\lambda: \lambda \in \mathbb{C}, \operatorname{Im} \lambda \leq 0\}$.

Therefore, $N^{+}$and $N^{-}$are analytic in $\mathbb{C}_{+}=\{\lambda: \lambda \in \mathbb{C}, \operatorname{Im} \lambda>0\}$ and $\mathbb{C}_{-}=\{\lambda: \lambda \in \mathbb{C}, \operatorname{Im} \lambda<$ $0\}$, respectively, and are continuous up to the real axis. The functions $N^{+}$and $N^{-}$are called Jost functions of $L$.

\section{Eigenvalues and spectral singularities of $L$}

We will denote the set of all eigenvalues and spectral singularities of $L$ by $\sigma_{d}(L)$ and $\sigma_{s s}(L)$, respectively. It is evident that

$$
\begin{aligned}
& \sigma_{d}(L)=\left\{\lambda: \lambda \in \mathbb{C}_{+}, N^{+}(\lambda)=0\right\} \cup\left\{\lambda: \lambda \in \mathbb{C}_{-}, N^{-}(\lambda)=0\right\}, \\
& \sigma_{s s}(L)=\left\{\lambda: \lambda \in \mathbb{R}^{*}, N^{+}(\lambda)=0\right\} \cup\left\{\lambda: \lambda \in \mathbb{R}^{*}, N^{-}(\lambda)=0\right\}, \\
& \left\{\lambda: \lambda \in \mathbb{R}^{*}, N^{+}(\lambda)=0\right\} \cap\left\{\lambda: \lambda \in \mathbb{R}^{*}, N^{-}(\lambda)=0\right\}=\emptyset,
\end{aligned}
$$

where $\mathbb{R}^{*}=\mathbb{R} \backslash\{0\}$.

Definition 1 The multiplicity of a zero of $N^{+}\left(\right.$or $\left.N^{-}\right)$in $\overline{\mathbb{C}}_{+}\left(\right.$or $\left.\overline{\mathbb{C}}_{-}\right)$is called the multiplicity of the corresponding eigenvalue or spectral singularity of $L$.

From (3.1) we find that, in order to investigate the quantitative properties of the eigenvalues and the spectral singularities of $L$, we need to discuss the quantitative properties of the zeros of $N^{+}$and $N^{-}$in $\overline{\mathbb{C}}_{+}$and $\overline{\mathbb{C}}_{-}$, respectively.

Let

$$
M_{1}^{ \pm}:=\left\{\lambda: \lambda \in \mathbb{C}_{ \pm}, N^{ \pm}(\lambda)=0\right\}, \quad M_{2}^{ \pm}:=\left\{\lambda: \lambda \in \mathbb{R}^{*}, N^{ \pm}(\lambda)=0\right\} .
$$

Let us denote the set of all limit points of $M_{1}^{+}$and $M_{1}^{-}$by $M_{3}^{+}$and $M_{3}^{-}$and the set of all zeros of $N^{+}$and $N^{-}$with infinite multiplicity in $\overline{\mathbb{C}}_{+}$and $\overline{\mathbb{C}}_{-}$, by $M_{4}^{+}$and $M_{4}^{-}$, respectively. 
It follows from the boundary uniqueness theorem of analytic functions that

$$
M_{3}^{ \pm} \subset M_{2}^{ \pm}, \quad M_{4}^{ \pm} \subset M_{2}^{ \pm}, \quad M_{3}^{ \pm} \subset M_{4}^{ \pm},
$$

and the linear Lebesgue measures of $M_{3}^{ \pm}$and $M_{4}^{ \pm}$are zero.

Using (3.1) and (3.2), we get

$$
\sigma_{d}(L)=M_{1}^{+} \cup M_{1}^{-}, \quad \sigma_{s s}(L)=M_{2}^{+} \cup M_{2}^{-} .
$$

Now, let us suppose that

$$
q, q^{\prime} \in \mathrm{AC}\left(\mathbb{R}_{+}\right), \quad \lim _{x \rightarrow \infty}|q(x)|+\left|q^{\prime}(x)\right|=0, \quad \int_{0}^{\infty} x^{5}\left|q^{\prime \prime}(x)\right| d x<\infty .
$$

Theorem 1 Under condition (3.5) the functions $N^{+}$and $N^{-}$have the following representations:

$$
\begin{array}{ll}
N^{+}(\lambda)=i \alpha_{2} \lambda^{3}+\beta^{+} \lambda^{2}+\delta^{+} \lambda+\varphi^{+}+\int_{0}^{\infty} f^{+}(t) e^{i \lambda t} d t, \quad \lambda \in \overline{\mathbb{C}}_{+}, \\
N^{-}(\lambda)=i \alpha_{2} \lambda^{3}+\beta^{-} \lambda^{2}+\delta^{-} \lambda+\varphi^{-}+\int_{0}^{\infty} f^{-}(t) e^{-i \lambda t} d t, \quad \lambda \in \overline{\mathbb{C}}_{-}
\end{array}
$$

where $\beta^{ \pm}, \delta^{ \pm}, \varphi^{ \pm} \in \mathbb{C}$, and $f^{ \pm} \in L_{1}\left(\mathbb{R}_{+}\right)$.

Proof Using (2.3), (2.4), and (2.6) we have (3.6), where

$$
\begin{aligned}
& \beta^{+}=i \alpha_{1}-\alpha_{2} K(0,0)-\beta_{2}, \\
& \delta^{+}=i \alpha_{0}+i \alpha_{2} K_{x}(0,0)-\alpha_{1} K(0,0)-\beta_{1}-i \beta_{2} K(0,0), \\
& \varphi^{+}=-\alpha_{2} K_{x t}(0,0)+i \alpha_{1} K_{x}(0,0)-\alpha_{0} K(0,0)-\beta_{0}+\beta_{2} K_{t}(0,0), \\
& f^{+}(t)=-\alpha_{2} K_{x t t}(0, t)+i \alpha_{1} K_{x t}(0, t)+\alpha_{0} K_{x}(0, t)+\beta_{2} K_{t t}(0, t)-i \beta_{1} K_{t}(0, t)-\beta_{0} K(0, t) .
\end{aligned}
$$

The following result is obtained in [23]:

$$
\left|K_{t t}(0, t)\right| \leq c\left[t\left|q\left(\frac{t}{2}\right)\right|+\left|q^{\prime}\left(\frac{t}{2}\right)\right|+t w\left(\frac{t}{2}\right)+w_{1}\left(\frac{t}{2}\right)\right] .
$$

Then from (2.4), (2.5), and (3.9), we get

$$
\begin{aligned}
\left|K_{x t t}(0, t)\right| \leq & c\left[\left|q^{\prime \prime}\left(\frac{t}{2}\right)\right|+t\left|q^{\prime}\left(\frac{t}{2}\right)\right|+t^{2}\left|q\left(\frac{t}{2}\right)\right|\right. \\
& \left.+t^{2} \sigma\left(\frac{t}{2}\right)+t \sigma_{1}\left(\frac{t}{2}\right)+\delta\left(\frac{t}{2}\right)+\delta_{1}\left(\frac{t}{2}\right)\right]
\end{aligned}
$$

where

$$
\delta(x)=\int_{x}^{\infty}\left|q^{\prime}(s)\right| d s, \quad \delta_{1}(x)=\int_{x}^{\infty} \delta(t) d t
$$

and $c>0$ is a constant. 
It follows from (2.5), (3.8), (3.9), and (3.10) that $f^{+} \in L_{1}\left(\mathbb{R}_{+}\right)$. In a similar way we obtain (3.7).

Theorem 2 Under the condition (3.5), we have:

(i) The set of eigenvalues of $L$ is bounded, has at most a countable number of elements, and its limit points can lie only in a bounded subinterval of the real axis.

(ii) The set of spectral singularities of $L$ is bounded and $\mu\left(\sigma_{s s}(L)\right)=0$.

Proof From (3.8), (3.9), and (3.10), we have

$$
\begin{array}{ll}
N^{+}(\lambda)=i \alpha_{2} \lambda^{3}+\beta^{+} \lambda^{2}+\delta^{+} \lambda+\varphi^{+}+o(1), & \lambda \in \overline{\mathbb{C}}_{+},|\lambda| \rightarrow \infty \\
N^{-}(\lambda)=i \alpha_{2} \lambda^{3}+\beta^{-} \lambda^{2}+\delta^{-} \lambda+\varphi^{-}+o(1), & \lambda \in \overline{\mathbb{C}}_{-},|\lambda| \rightarrow \infty
\end{array}
$$

Using (3.4), (3.11), and the uniqueness theorems of analytic functions [24], we obtain (i) and (ii).

\section{Theorem 3 If}

$$
q, q^{\prime} \in \mathrm{AC}\left(\mathbb{R}_{+}\right), \quad \lim _{x \rightarrow \infty}|q(x)|+\left|q^{\prime}(x)\right|=0, \quad \int_{0}^{\infty} e^{\varepsilon x}\left|q^{\prime \prime}(x)\right|<\infty, \quad \varepsilon>0
$$

then the operator L has a finite number of eigenvalues and spectral singularities, and each of them is of finite multiplicity.

Proof Using (2.5), (3.8), (3.9), (3.10), and (3.12) we find that

$$
\left|f^{+}(t)\right| \leq c e^{-\left(\frac{\varepsilon}{2}\right) t}
$$

where $c>0$ is a constant. By (3.6) and (3.13) we observe that the function $N^{+}$has an analytic continuation to the half-plane $\operatorname{Im} \lambda>-\frac{\varepsilon}{2}$. So we get $M_{4}^{+}=\emptyset$. It follows from (3.3) that $M_{3}^{+}=\emptyset$. Therefore the sets $M_{1}^{+}$and $M_{2}^{+}$have a finite number of elements with a finite multiplicity. We obtain similar results for the sets $M_{1}^{-}$and $M_{2}^{-}$. From (3.4) we have the proof of the theorem.

It is seen that the condition (3.12) guarantees the analytic continuation of the functions $N^{+}$and $N^{-}$from the real axis to the lower and upper half-planes, respectively. So the finiteness of eigenvalues and spectral singularities of $L$ are achieved as a result of this analytic continuation.

Now let us suppose that

$$
q, q^{\prime} \in \mathrm{AC}\left(\mathbb{R}_{+}\right), \quad \lim _{x \rightarrow \infty}|q(x)|+\left|q^{\prime}(x)\right|=0, \quad \sup _{x \in \mathbb{R}_{+}}\left[e^{\varepsilon \sqrt{x}}\left|q^{\prime \prime}(x)\right|\right]<\infty, \quad \varepsilon>0
$$

which is weaker than (3.12).

It is evident that under the condition (3.14) the function $N^{+}$is analytic in $\mathbb{C}_{+}$and infinitely differentiable on the real axis. But $N^{+}$does not have an analytic continuation from the real axis to the lower half-plane. Similarly, $N^{-}$does not have an analytic continuation from the real axis to the upper half-plane, either. Therefore, under the condition (3.14) the 
finiteness of eigenvalues and spectral singularities of $L$ cannot be proved in a way similar to Theorem 3.

Lemma 4 If (3.14) holds, then $M_{4}^{+}=M_{4}^{-}=\emptyset$.

Proof It follows from (3.6) and (3.14) that the function $N^{+}$is analytic in $\mathbb{C}_{+}$, and all of its derivatives are continuous up to the real axis. Moreover, by Theorem 2 for sufficiently large $T>0$, we have

$$
\left|\int_{-\infty}^{-T} \frac{\operatorname{In}\left|N^{+}(\lambda)\right|}{1+\lambda^{2}} d \lambda\right|<\infty, \quad\left|\int_{T}^{\infty} \frac{\operatorname{In}\left|N^{+}(\lambda)\right|}{1+\lambda^{2}} d \lambda\right|<\infty
$$

From (3.6), we obtain

$$
\left|\frac{d^{n}}{d \lambda^{n}} N^{+}(\lambda)\right| \leq A_{n}^{+}, \quad \lambda \in \overline{\mathbb{C}}_{+},|\lambda| \leq 2 T, n \in \mathbb{N} \cup\{0\}
$$

where

$$
A_{n}^{+}=2^{n} c \int_{0}^{\infty} t^{n} e^{-\left(\frac{\varepsilon}{2}\right) \sqrt{t}} d t, \quad n \in \mathbb{N} \cup\{0\},
$$

and $c>0$ is a constant. Since the function $N^{+}$is not equal to zero identically, by Pavlov's theorem [25], $M_{4}^{+}$satisfies

$$
\int_{0}^{h} \ln T^{+}(s) d \mu\left(M_{4}^{+}, s\right)>-\infty
$$

where $T^{+}(s)=\inf _{n} \frac{A_{n}^{+} s^{n}}{n !}, \mu\left(M_{4}^{+}, s\right)$ is the linear Lebesgue measure of an $s$-neighborhood of $M_{4}^{+}$, and the constant $A_{n}^{+}$is defined by (3.15).

Now we obtain the following estimates for $A_{n}^{+}$:

$$
A_{n}^{+} \leq A a^{n} n ! n^{n},
$$

where $A$ and $a$ are constants depending on $c$ and $\varepsilon$. Substituting (3.17) in the definition of $T^{+}(s)$, we arrive at

$$
T^{+}(s) \leq A \inf _{n}\left(a^{n} s^{n} n^{n}\right) \leq A \exp \left(-a^{-1} e^{-1} s^{-1}\right) .
$$

Now by (3.16) we get

$$
\int_{0}^{h} \frac{1}{s} d \mu\left(M_{4}^{+}, s\right)<\infty .
$$

Inequality (3.18) holds for arbitrary $s$ if and only if $\mu\left(M_{4}^{+}, s\right)=0$ or $M_{4}^{+}=\emptyset$. In a similar way we can prove that $M_{4}^{-}=\emptyset$.

Theorem 5 Under the condition (3.14) the operator L has a finite number of eigenvalues and spectral singularities, and each of them is of a finite multiplicity. 
Proof To be able to prove the theorem, we have to show that the functions $N^{+}$and $N^{-}$ have a finite number of zeros with finite multiplicities in $\overline{\mathbb{C}}_{+}$and $\overline{\mathbb{C}}_{-}$, respectively. We give the proof for $N^{+}$.

It follows from (3.3) and Lemma 4 that $M_{3}^{+}=\emptyset$. So the bounded sets $M_{1}^{+}$and $M_{2}^{+}$have no limit points, i.e., the function $N^{+}$has only a finite number of zeros in $\overline{\mathbb{C}}_{+}$. Since $M_{4}^{+}=\emptyset$, these zeros are of finite multiplicity.

\section{Competing interests}

The authors declare that they have no competing interests.

\section{Authors' contributions}

All authors contributed equally and significantly in writing this paper. All authors read and approved the final manuscript.

\section{Author details}

${ }^{1}$ Department of Mathematics, Karamanoglu Mehmetbey University, Karaman, 70100, Turkey. ${ }^{2}$ Department of Mathematics, Kastamonu University, Kastamonu, 37100, Turkey.

\section{Acknowledgements}

The authors would like to express their thanks to the reviewers for their helpful comments and suggestions.

Received: 5 September 2014 Accepted: 14 January 2015 Published online: 03 February 2015

\section{References}

1. Naimark, MA: Investigation of the spectrum and the expansion in eigenfunctions of a non-selfadjoint operator of second order on a semi-axis. Am. Math. Soc. Transl. (2) 16, 103-193 (1960)

2. Lyance, VE: A differential operators with spectral singularities I. Am. Math. Soc. Transl. (2) 60, 185-225 (1967)

3. Lyance, VE: A differential operators with spectral singularities II. Am. Math. Soc. Transl. (2) 60, 227-283 (1967)

4. Adıvar, M: Quadratic pencil of difference equations: Jost solutions, spectrum, and principal vectors. Quaest. Math. 33, 305-323 (2010)

5. Krall, AM: A nonhomogeneous eigenfunction expansion. Trans. Am. Math. Soc. 117, 352-361 (1965)

6. Krall, AM: The adjoint of differential operator with integral boundary conditions. Proc. Am. Math. Soc. 16, 738-742 (1965)

7. Krall, AM, Bairamov, E, Cakar, O: Spectrum and spectral singularities of a quadratic pencil of a Schrödinger operator with a general boundary conditions. J. Differ. Equ. 151, 252-267 (1999)

8. Krall, AM, Bairamov, E, Cakar, O: Spectral analysis of non-selfadjoint discrete Schrödinger operators with spectral singularities. Math. Nachr. 231, 89-104 (2001)

9. Bairamov, E, Cakar, O, Krall, AM: An eigenfunction expansion for a quadratic pencil of a Schrödinger operator with spectral singularities. J. Differ. Equ. 151, 268-289 (1999)

10. Bairamov, E, Cakar, O, Krall, AM: Non-selfadjoint difference operators and Jacobi matrices with spectral singularities. Math. Nachr. 229, 5-14 (2001)

11. Bairamov, E, Celebi, AO: Spectrum and spectral expansion for the non-selfadjoint discrete Dirac operators. Q. J. Math. 50, 371-384 (1999)

12. Adıvar, M, Bairamov, E: Spectral singularities of the nonhomogeneous Sturm-Liouville equations. Appl. Math. Lett. 15 825-832 (2002)

13. Fulton, CT: Two-point boundary value problems with eigenvalue parameter contained in the boundary conditions. Proc. R. Soc. Edinb. A 77, 293-308 (1977)

14. Chernozhukova, AY, Freiling, G: A uniqueness theorem for inverse spectral problems depending nonlinearly on the spectral parameter. Inverse Probl. Sci. Eng. 17, 777-785 (2009)

15. Freiling, G, Yurko, VA: Inverse problems for Sturm-Liouville equations with boundary conditions polynomially dependent on the spectral parameter. Inverse Probl. 26(6), 055003 (2010)

16. Binding, PA, Browne, PJ, Watson, BA: Transformations between Sturm-Liouville problems with eigenvalue dependent and independent boundary conditions. Bull. Lond. Math. Soc. 33, 749-757 (2001)

17. Binding, PA, Browne, PJ, Watson, BA: Sturm-Liouville problems with boundary conditions rationally dependent on the eigenparameter I. Proc. Edinb. Math. Soc. 33, 749-757 (2001)

18. Binding, PA, Browne, PJ, Watson, BA: Sturm-Liouville problems with boundary conditions rationally dependent on the eigenparameter II. Comput. Appl. Math. 148, 147-168 (2002)

19. Code, WJ, Browne, PJ: Sturm-Liouville problems with boundary conditions depending quadratically on the eigenparameter. J. Math. Anal. Appl. 309, 729-742 (2005)

20. Yokus, N: Principal functions of non-selfadjoint Sturm-Liouville problems with eigenvalue-dependent boundary conditions. Abstr. Appl. Anal. 2011, Article ID 358912 (2011)

21. Bairamov, E, Koprubasi, T: Eigenparameter dependent discrete Dirac equations with spectral singularities. Appl. Math. Comput. 215, 4216-4220 (2010)

22. Marchenko, VA: Sturm-Liouville Operators and Applications. Birkhäuser, Basel (1986)

23. Bairamov, E, Yokus, N: Spectral singularities of Sturm-Liouville problems with eigenvalue-dependent boundary conditions. Abstr. Appl. Anal. 2009, Article ID 28959 (2009)

24. Dolzhenko, EP: Boundary value uniqueness theorems for analytic functions. Math. Notes 26(6), 437-442 (1979)

25. Pavlov, BS: On separation conditions for spectral components of a dissipative operator. Math. USSR, IzV. 9, $113-137$ (1975) 